Saudi Journal of Oral and Dental Research

Abbreviated Key Title: Saudi J Oral Dent Res

ISSN 2518-1300 (Print) |ISSN 2518-1297 (Online)

Scholars Middle East Publishers, Dubai, United Arab Emirates

Journal homepage: https://saudijournals.com/sjodr

\title{
A Comparitive Evaluation of Apical Microleakage of Two Retrograde Filling Materials "A Stereo Microscopic Study"
}

Dr. S.K.Yamini Parimala, Dr. S.Sunil Kumar*, Dr. S. Datta Prasad, Dr. C. Sunil Kumar, Dr. N. Vamsee Krishna, Dr. K.S. Chandra Babu

Department of Conservative Dentistry and Endodontics, C.K.S. Theja Institute of Dental Sciences and Research, Chadalawada Nagar, Renigunta Road, Tirupati

DOI: $10.36348 /$ sjodr.2020.v05i02.004

| Received: 01.02.2020 | Accepted: 08.02.2020 | Published: 12.02.2020

*Corresponding author: Dr. S.Sunil Kumar

\section{Abstract}

The prime concept behind root canal treatment is to achieve a proper coronal and apical seal. Apicoectomy (root-end resection) with retrograde filling is a widely followed procedure to encounter the condition when all the efforts of endodontic treatment have failed. Among various ideal requirements of a root-end filling material, proper sealing ability is one of the most required properties. So, the selection of appropriate root-end filling material is to be done to prevent microleakage in the apical area after root-end resection. This study aims to evaluate the apical microleakage of two rootend filling materials. "Biodentine," which is known for its excellent biocompatibility and "MTA Repair HP," which has superior handling properties are compared in this study. Root canal treatment till obturation is done for all the samples. Root-end resection is done by removal of the apical $3 \mathrm{~mm}$ with an angulation of $90^{\circ}$ to the long axis of the tooth. The root end cavity of a depth $3 \mathrm{~mm}$ is made. The samples were randomly divided into two groups, group 1 - MTA Repair HP, group 2 - Biodentine. Materials were manipulated according to the manufacturer's instructions, and the root end cavities were filled with the respective materials of the belonging groups. Two coats of nail varnish is applied, and the samples were immersed in methylene blue dye. Dye penetration study was done, and specimens wereexamined under a stereomicroscope. Results are tabulated and statistically analyzed. Within the limitations of the study, it is concluded that, in comparison to these two root-ends filling materials, Biodentine showed the highest sealing ability and less microleakage compared to MTA Repair HP, which showed moderate microleakage.

Keywords: Microleakage, Root end infection, Apicoectomy, Retrograde filling, apical seal, Biodentine, MTA Repair HP.

Copyright @ 2020: This is an open-access article distributed under the terms of the Creative Commons Attribution license which permits unrestricted use, distribution, and reproduction in any medium for non-commercial use (NonCommercial, or CC-BY-NC) provided the original author and source are credited.

\section{INTRODUCTION}

The main aim of the retrograde filling is to provide an apical seal, thereby preventing the egress of microbes and their toxins from the canal space into peri radicular tissues. Resection of root end and filling the canals does not accomplish this treatment goal. The main purpose of endodontic surgery is to achieve an impervious seal at the apex to eliminate bacterial contamination of the peri radicular tissues and encourage their regeneration.

Hermetically sealing all the pathways of communication between the pulpal and peri radicular tissues should be taken into consideration. Proper obturation and restoration of the tooth is an essential part of root canal therapy. Root canals must be sealed both coronally and apically to prevent leakage of oral fluids to avoid the recontamination of the canals.
When all efforts for the successful completion of orthograde endodontic therapy have failed, when there is a failure of non-surgical endodontic treatment or retreatment, then there will be a need for endodontic surgery to save the tooth. The root-end filling materials should be biocompatible and must provide an adequate seal to prevent leakage of irritants and microbes from root canals [1].

Many materials have been advocated for rootend filling till now in dental history. These include gold foil, resin composite, gutta-percha (GP), amalgam, glass ionomer, Super-ethoxy benzoic acid (Super EBA), and intermediate restorative material (IRM). Knowledge of the effect of root-end filling materials on the surrounding tissues is crucial as retrograde filling materials intimately contact peri radicular tissues [2]. 
Many efforts are being made to evaluate the apical sealing ability of many root-end filling materials. MTA was developed at Loma Linda University in 1993. Superior qualities of MTA regarding marginal adaptation were proved by the studies done by Torabinejad et al. and Fischer et al. It is established to seal all the pathways which are present between the root canal and peri radicular tissues. But Studies have also shown that MTA has poor handling properties [3].

To overcome the drawbacks of MTA, few materials have been introduced. These include BIODENTINE and MTA Repair HP. Both the materials possess their advantages compared to MTA. So the main aim of this study is to evaluate the apical microleakage of these tooth materials when used for root-end filling using the dye penetration method under the stereomicroscope.

\section{MATERIALS AND METHODS Sample preparation}

24 extracted maxillary central incisors with completely formed apices and straight canals were chosen for the study and were divided into two groups of 12 for performing the study. The crown and root surfaces were cleaned to remove any tissue remnants and debris. Preoperative radiographs were taken to rule out any calcifications/microcracks, etc., Decoronation of all the samples was done to maintain a standardized root length of $15 \mathrm{~mm}$ measuring from the root apex. Access cavities were made. Canal patency was checked using a 15 size $\mathrm{k}$ file. Canal preparation was done using hand $\mathrm{k}$ files till 50 size of apical enlargement. 3\% sodium hypochlorite and 17\% EDTA were used for canal irrigation. RCHelp was used as a lubricant between each subsequent filing. Canals were dried using absorbent paper points, and Canals were obturated with gutta-percha by lateral compaction technique. The access cavities were sealed with glass ionomer cement. After $24 \mathrm{hrs}$, the teeth were then stored in normal saline at $37^{\circ} \mathrm{c}$ and $100 \%$ humidity for one week.

\section{Apicoectomy and root-end filling procedures}

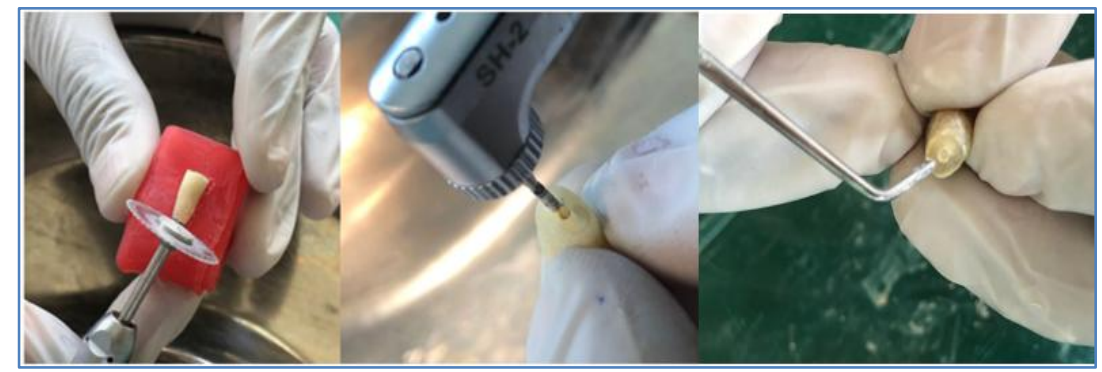

Fig-1: Procedure of apicoectomy and rootend filling

Apical $3 \mathrm{~mm}$ of the roots were resected at an angle of $90^{\circ}$ perpendiculars to the long axis of the tooth using a diamond disc. A $3 \mathrm{~mm}$ deep retrograde cavity was prepared using straight fissure diamond bur, and the prepared cavities were irrigated with saline and blot dried(fig.1). The teeth were randomly divided into two groups of 12 specimens each:

Group I: Mineral Trioxide Aggregate Repair HP (MTA Repair HP)

\section{Group II: Biodentine}

These materials were manipulated according to the manufacturer's instructions, and the root end filling is done (fig.1). The specimens were then coated with three coats of nail varnish except at the apical $1 \mathrm{~mm} \&$ then were allowed to dry.

The specimens were then suspended in $1 \%$ methylene blue for 72 hours. Following this, the teeth were rinsed for 15 minutes under running water. The teeth were then sectioned longitudinally, and the dye penetration was examined under stereomicroscope \& microleakage was evaluated in millimeters.

\section{Stereo microscopic images GROUP I (BIODENTINE)}

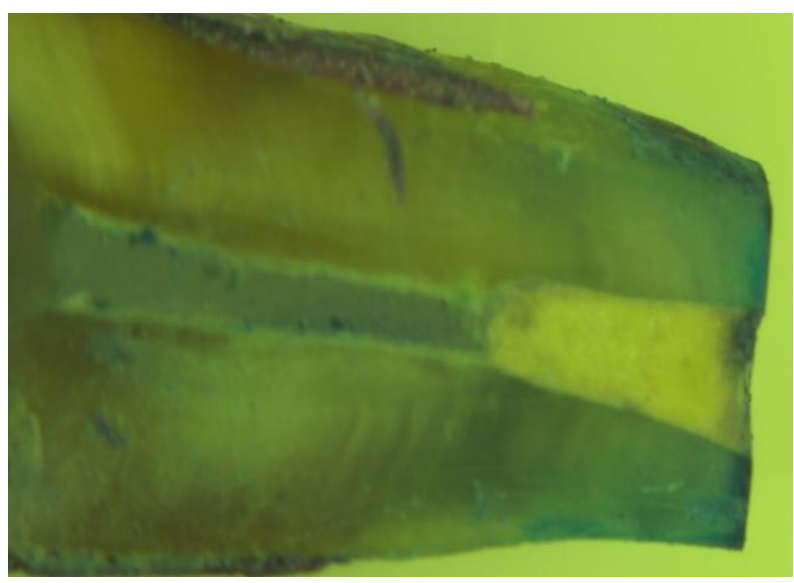

Fig-2: Biodentine with minimum leakage 


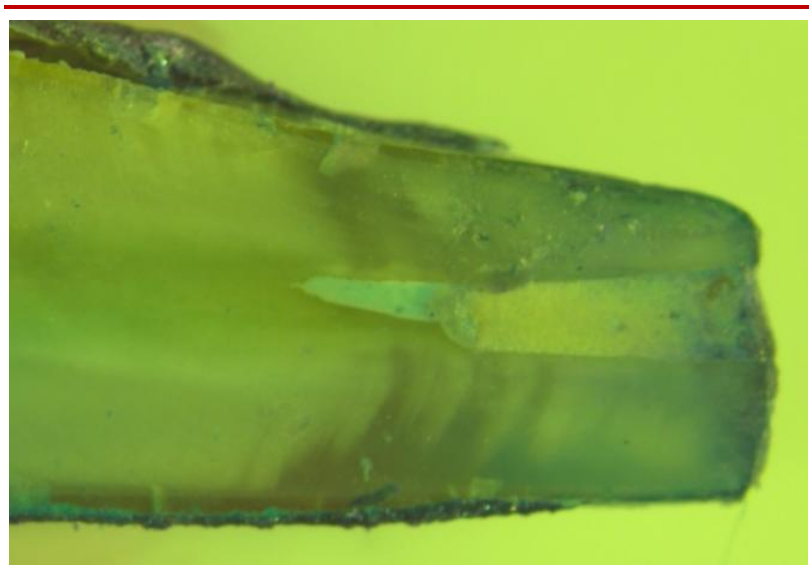

Fig-3: Biodentine with maximum leakage

\section{GROUP I (MTA REPAIR HP)}

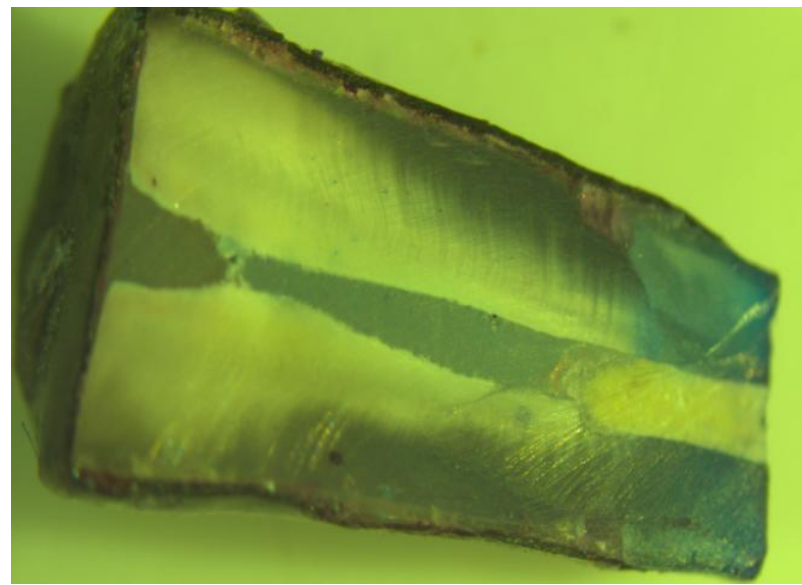

Fig-4: MTA REPAIR HP with minimum leakage

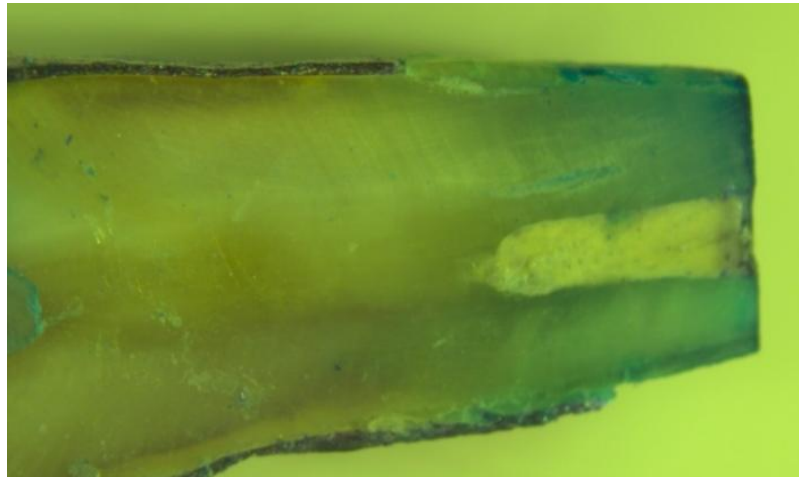

Fig-5: MTA REPAIR HP with maximum leakage

\section{RESULTS}

Leakage values for two different groups were measured. Student's t-test was used for statistical analysis. An average leakage value of $0.26 \mathrm{~mm}$ was seen with biodentine, and $0.48 \mathrm{~mm}$ was seen with MTA Repair HP.
Table-1: Mean apical micro leakage values

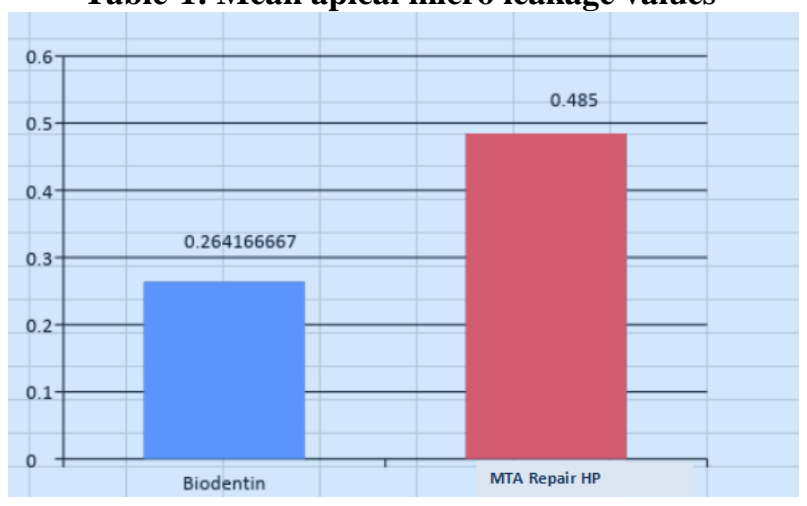

Mean wise graphical representation of the average microleakage of biodentine and MTA Repair HP was figured in which Biodentine showed the least microleakage (Ttable.1).

\section{DISCUSSION}

Localized fragmentation of the hertwing's epithelial root sheath develops, and this leaves a small gap, and blood vessels running through the dental papilla from the dental sac persist, resulting in the formation of lateral and apical ramifications. In this specific area, Dentinogenesis does not occur, giving rise to a canal containing small blood vessels and sometimes nerves. Although ramifications consist of connective tissue and blood vessels, this is not usually regarded as collateral blood supply and consequently provides a little contribution, if any, to pulp function, except possibly for the apical ramifications at apical 1-2 $\mathrm{mm}$ of the canal. Along the length of the root, Ramifications can be observed anywhere, but they occur more commonly in the apical portion [4].

Ramifications provide potential pathways through which bacteria and their products from the necrotic root canal might reach the periodontal ligament and cause disease. Likewise, bacteria from periodontal pockets might enter the pulp. Lateral canals and apical ramifications are difficult to reach, disinfect, clean, and fill during endodontic treatment. Their clinical significance has gained the attention of clinicians and researchers if these ramifications should be approached and also about the fate of the tissue present after treatment [4].

Based on these assumptions, successful endodontic therapy is accepted by the idea that lateral canals and apical ramifications should be filled. It is stated that the reason behind endodontic failures is the presence of lateral canals and apical ramifications if they are sufficiently large to harbor significant numbers of bacteria and to provide these bacteria with direct access to the periradicular tissues. Therefore, disinfection of apical ramifications and lateral canals in conditions of pulp necrosis and apical and lateral periodontitis should be considered an essential goal of 
the treatment, although challenging to achieve with current treatment procedures. If that is not possible other treatment options, if necessary, are to be considered. Bacteria located in lateral canals and apical ramifications can undoubtedly influence the outcome, it seems advisable to apply therapeutic plans to reach and incorporate these areas in the disinfection process [4].

In this study, apical $3 \mathrm{~mm}$ of the root is resected to mimic the procedure of apicoectomy. The main reason behind this is, an anatomical study was done on the root apex, which showed that at least $3 \mathrm{~mm}$ of the root-end must be removed to reduce $98 \%$ of apical ramifications and $93 \%$ of lateral canals [5]. The amount of root resection which satisfies the biological principles was a question. Gilheany 1994 suggested that bacterial leakage from the canal can be minimized by removal of at least $2 \mathrm{~mm}$ [5]. In support of this, many studies have been done and concluded that it is essential to remove at least $3 \mathrm{~mm}$ of the root end [6].

Jaiane Bandoli Monteiro did a study in evaluating the Effect of Resection Angle on Stress Distribution after Root- End Surgery. In this, a slight stress concentration could be observed in the cement line at its initial part of the third cervical portion in the models without resection and with $45^{\circ}$ angle resection, while with $90^{\circ}$ resection, a better stress distribution occurred. They concluded that in situations where root resection is needed, 90-degree resection could improve stress distribution. This behavior can be explained by the larger surface area of the dental structure to receive the tensile stress [7].

The microleakage evaluation was done using the dye penetration method using $1 \%$ methylene blue. Due to its simplicity and cost-effectiveness. The dye penetration test is considered one of the most popular techniques used in endodontic leakage studies [8].

Fig.2 shows the stereomicroscopic image of biodentine with minimum microleakage with in the test group and fig. 3 shows the stereomicroscopic image of biodentine with maximum microleakage wiyh in the test group.

In conclusion, drawn from a study done by $\mathrm{K}$, M. F. AHLBERG in comparing the apical dye penetration patterns shown by methylene blue and India ink in root-filled teeth, methylene blue, penetrated more deeply along root canal fillings because of its low molecular weight than India ink with a large particle zsize [9].

The first group used Biodentine as root-end filling material, which is with Active Biosilicate Technology was announced by dental materials manufacturer Septodont in September of 2010, and made available in January of 2011. According to the manufacturer, the material can be used as a "dentine replacement material whenever original dentine is damaged."

Fig.4 shows the stereomicroscopic image of MTA Repair HP with minimum microleakage with in the test group and fig.5 shows the stereomicroscopic image of MTA Repir HP with maximum microleakage wiyh in the test group

Group II MTA Repair HP is a Bioceramic reparative cement with high plasticity. MTA (Mineral Trioxide Aggregate) has its extensive use in Endodontics due to the clinical results proven through numerous scientific studies. However, due to the physical properties inherent to the powder of the product, the "sandy" consistency hinders the manipulation and transport of the material to the site of repair.

To improve these characteristics, ANGELUS has developed a new formulation called "MTA REPAIR HP - High Plasticity" MTA. This unique formula maintains all the chemical and biological properties of the original MTA, which guarantees the success of the treatment but changes its physical properties of manipulation. The result is a product with greater plasticity, facilitating manipulation and insertion in the dental cavity [10].

It is a Restorative cement ready for use. MTA REPAIR HP is endodontic restorative cement with high plasticity, composed of mineral oxides in the form of fine hydrophilic particles. It is indicated for cases of root perforation (canal and furcation), iatrogenic or by caries, root perforation by internal resorption, retro filling, direct pulp capping, pulpotomy, apexogenesis, and apexification[10]. It mainly constitutes Tricalcium silicate 3CaO.SiO2; Dicalcium silicate 2CaO.SiO2; Tricalcium aluminate 3CaO.Al2O3; Calcium oxide $\mathrm{CaO}$; Calcium Tungstate $\mathrm{CaWO} 4$ in its powder and Water and Plasticizers in its liquid.

MTA REPAIR HP solidifies when kept in a wet environment after spatulation. Initial setting time is approximately 15 minutes; Radiopacity Nearly matches that of gutta-percha and is more radiopaque than dentine and bone; a clinical advantage is the absence of dental discoloration due to the usage of CaWO4 as radio opacifier.

The main advantage over other materials is that the particle size after hydration allows for easy manipulation and insertion into the dental cavity, Low solubility, Excellent marginal sealing capacity through controlled setting expansion, which impedes the migration of microorganisms and fluids to the inside of the root canal. It also shows excellent biological sealing of root perforation (channel and furcation) to induce the formation of periradicular cement. It also promotes the formation of a dent in bridge when used in pulp 
capping. It allows for use in wet medium without change of its properties.

MTA Repair HP has superior marginal sealing ability same as resulting from its hydrophilic properties and formation of the interfacial layer between material and dentin. It was found that further hydration of MTA powder by moisture can result in an increase in compressive strength and decreased leakage.

Because the MTA mixture is a sand-like paste, it is difficult to place in the prepared root-end cavities. In addition to these handling difficulties, freshly mixed MTA can wash out if exposed to excessive fluids due to its long setting time having a detrimental effect on its sealing ability. This is overcome by MTA Repair HP by its improved consistency.

Biodentine is a retrograde and repair material launched in capsule form [11]. Biodentine (Septodont, Saint Maur des Fosses, France) powder is mainly composed of tricalcium silicate, calcium carbonate, and zirconium oxide as the radio-pacifier, while Biodentine liquid contains calcium chloride as the setting accelerator and water as reducing agent [11].

This material uses zirconium oxide to give radiopacity. Contrary to bismuth oxide, zirconium oxide was shown to possess biocompatible characteristics and indicated as a bioinert material with high mechanical properties and favorable resistance to corrosion [12].

From a biomechanical point of view, the sealing properties of Biodentine have been reported to be superior to MTA. The formation of mineral tags was similar to those observed with MTA along with resistance to acid degradation, as noted in inflammatory sites. The main difference between Biodentine and commercially available MTA calcium silicates is the absence of calcium aluminates and calcium sulfate in the formulation, which are known to bring decreased mechanical strength as well as longer setting time [12].

The primary clinical advantage of Biodentine is its fast setting (between 12 and $15 \mathrm{~min}$ ). This is an advantage when compared to the $170 \mathrm{~min}$ of MTA

All the factors mentioned above justify the results of this study, which shows the excellent sealing property of biodentine with the least microleakage values compared to MTA Repair HP.

\section{CONCLUSION}

Within the limitations of this in vitro study, it has been concluded that in comparison to these two root-end filling materials, group II - Biodentine showed the highest sealing ability and least microleakage compared to group I - MTA Repair HP which showed moderate leakage.

\section{REFERENCES}

1. Anil, K.T., Nitish, M. (2018). Root End Filling Materials - A Review. Scholars Journal of Dental Sciences. Sept 9(5): 435-437

2. Abusrewil, S. M., McLean, W., \& Scott, J. A. (2018). The use of Bioceramics as root-end filling materials in periradicular surgery: A literature review. The Saudi dental journal, 30(4), 273-282.

3. Shetty, S., Hiremath, G., \& Yeli, M. (2017). A comparative evaluation of sealing ability of four root end filling materials using fluid filtration method: An in vitro study. Journal of conservative dentistry: JCD, 20(5), 307.

4. Ricucci, D., \& Siqueira Jr, J. F. (2010). Fate of the tissue in lateral canals and apical ramifications in response to pathologic conditions and treatment procedures. Journal of endodontics, 36(1), 1-15.

5. Ma, X., Li, C., Jia, L., Wang, Y., Liu, W., Zhou, X., \& Huang, D. (2016). Materials for retrograde filling in root canal therapy. Cochrane Database of Systematic Reviews, (12).

6. Stropko, J. J., Doyon, G. E., \& Gutmann, J. L. (2005). Root-end management: resection, cavity preparation, and material placement. Endodontic Topics, 11(1), 131-151.

7. Monteiro, J. B., Dal Piva, A. M. D. O., Tribst, J. P. M., Borges, A. L. S., \& Tango, R. N. (2018). The effect of resection angle on stress distribution after root-end surgery. Iranian endodontic journal, 13(2), 188.

8. Chhaparwal, S., Ballal, N. V., Menezes, N. D., \& Kamath, S. U. (2017). Effect of chelating agents on sealing ability of Biodentine and mineral trioxide aggregate. Saudi Endodontic Journal, 7(1), 16.

9. Ahlberg, K. M. F., Assavanop, P., \& Tay, W. M. (1995). A comparison of the apical dye penetration patterns shown by methylene blue and India ink in root-filled teeth. International Endodontic Journal, 28(1), 30-34.

10. MTA REPAIR HP. Available from http://angelusdental.com/img/arquivos/mta_repair_ hp_bula.

11. Saxena, P., Gupta, S. K., \& Newaskar, V. (2013). Biocompatibility of root-end filling materials: recent update. Restorative dentistry \& endodontics, 38(3), 119-127.

12. Caron, G., Azérad, J., Faure, M. O., Machtou, P., \& Boucher, Y. (2014). Use of a new retrograde filling material (Biodentine) for endodontic surgery: two case reports. International journal of oral science, 6(4), 250-253.

13. Tanalp, J., Karapınar-Kazandağ, M., Dölekoğlu, S., \& Kayahan, M. B. (2013). Comparison of the radiopacities of different root-end filling and repair materials. The Scientific World Journal, 2013. 